PROCEEDINGS OF THE AMERICAN MATHEMATICAL SOCIETY

Volume 127, Number 7, Pages 1893-1902

S 0002-9939(99)04662-6

Article electronically published on February 26, 1999

\title{
SPECIAL-VALUED SUBGROUPS OF LATTICE-ORDERED GROUPS
}

\author{
YUANQIAN CHEN AND PAUL CONRAD
}

(Communicated by Lance W. Small)

\begin{abstract}
We prove that the intersection of all maximal special-valued subgroups of a lattice-ordered group $G$ is the special-valued quasi-torsion radical of a lattice-ordered group $G$, which extends our earlier result that the intersection of all maximal finite-valued subgroups of a lattice-ordered group $G$ is the finite-valued torsion radical of $G$. We also show that the class $A_{f}$ of almost finite-valued lattice-ordered groups is a quasi-torsion class, and the $A_{f}$ quasi-torsion radical of a group is equal to the intersection of the group with the lateral completion of the finite-valued torsion radical of the group.
\end{abstract}

\section{INTRODUCTION}

For the basic definitions and results in lattice-ordered group theory, see M. Anderson and T. Feil [2] and M. Darnel [8]. A lattice-ordered group, written $\ell$-group, is a partially ordered group $(G, \leq)$ where the partial order is a lattice (meaning that each pair of elements $a, b$ of $G$ has a least upper bound $a \vee b$ and a greatest lower bound $a \wedge b$ ). An $\ell$-subgroup $A$ of an $\ell$-group $G$ is both a subgroup and a sublattice of $G$. $A$ is a convex $\ell$-subgroup of $G$ if $a, b \in A$ and $a \leq g \leq b$ imply that $g \in A$. A normal convex $\ell$-subgroup is an $\ell$-ideal. A convex $\ell$-subgroup which is maximal with respect to not containing some $g \in G$ is called regular and is a value of $g$. A regular subgroup $A$ is an essential value if it contains all the values for some $g \in G$. Element $g$ is special if it has a unique value and in this case the value is called a special value. Regular subgroups of $G$ form a root system under inclusion, written $\Gamma(G)$. (That is, $\Gamma(G)$ is a partially ordered set for which $\{\alpha \in \Gamma(G) \mid \alpha \geq \gamma\}$ is totally ordered, for any $\gamma \in \Gamma(G)$.) A subset $\Delta \subseteq \Gamma(G)$ is plenary if $\cap \Delta=\{0\}$ and $\Delta$ is a dual ideal in $\Gamma(G)$; that is, if $\delta \in \Delta, \gamma \in \Gamma(G)$ and $\gamma>\delta$, then $\gamma \in \Delta$. If $G$ is an abelian $\ell$-group, then $G$ is $\ell$-isomorphic to an $\ell$-subgroup of $V(\Gamma(G), R)$ such that if $\gamma$ is a value of $g \in G$, then $\gamma$ is a maximal component of $g$ after the embedding, where $V(\Gamma(G), R)$ is the abelian $\ell$-group of all real-valued functions $v$ on $\Gamma(G)$ for which $v(\gamma) \in R$ and the support of each $v$ satisfies the ascending chain condition. This is a consequence of the Conrad-Harvey-Holland embedding theorem for abelian lattice-ordered groups.

Received by the editors July 16, 1996 and, in revised form, September 2, 1997.

1991 Mathematics Subject Classification. Primary 06F15, 06F20; Secondary 20F60.

Key words and phrases. Torsion class and quasi-torsion class, finite-valued and special-valued subgroups of a lattice-ordered group. 
$\Sigma(\Delta, R)$ is the $\ell$-subgroup of $V(\Delta, R)$ containing all elements $v \in V$ with finite supports. $F(\Delta, R)$ is the $\ell$-subgroup of $V(\Delta, R)$ containing all elements $v \in V$ whose supports are contained in a finite number of chains in $\Delta$.

For any $g \in G, G(g)=\{h \in G|| h|\leq n| g \mid$, for some positive integer $n\}$, the principal convex $\ell$-subgroup of $G$ generated by $g$, is the least convex $\ell$-subgroup of $G$ that contains $g$.

An $\ell$-group $G$ is finite-valued if every element of $G$ has only a finite number of values; this is equivalent to the statement that every element of $G$ can be expressed as a finite sum of disjoint special elements. Each element of $G$ is also called finitevalued. An $\ell$-group $G$ is special-valued if $G$ has a plenary subset of special values; this is equivalent to the statement that each positive element of $G$ can be expressed as the join of a set of pairwise disjoint positive special elements. A positive element $g$ of $G$ is special-valued if $g$ can be expressed as the join of disjoint special elements.

A lattice homomorphism is complete if it preserves all (not necessarily finite) meets and joins. A convex $\ell$-subgroup is closed if it is closed with respect to infinite meets and joins which exist in the $\ell$-group. A convex $\ell$-subgroup $C$ of an $\ell$-group $G$ is closed if and only if the natural lattice homomorphism from $G$ onto its lattice $G / C$ of right cosets is complete. Extensions which preserve the lattice of closed convex $\ell$-subgroups are called $a^{*}$-extensions.

An $\ell$-group is laterally complete (conditionally laterally complete) if for any subset (bounded subset) $\left\{g_{\alpha} \mid \alpha \in A\right\}$ of disjoint positive elements, $\vee_{A} g_{\alpha}$ exists.

A torsion class is a class of lattice-ordered groups that is closed under convex $\ell$ subgroups, $\ell$-homomorphic images, and joins of convex $\ell$-subgroups. For an $\ell$-group $G$ and a torsion class $T, T(G)$ indicates the join of all the convex $\ell$-subgroups of $G$ that belong to T. $T(G)$ is then the largest convex $\ell$-subgroup of $G$ that belongs to $T$, called the torsion radical of $G$. A quasi-torsion class is a class of $\ell$-groups which is closed under convex $\ell$-subgroups, complete $\ell$-homomorphic images, and joins of convex $\ell$-subgroups. For an $\ell$-group $G$ and a quasi-torsion class $Q, Q(G)$ indicates the join of all the convex $\ell$-subgroups of $G$ that belong to $Q . Q(G)$ is then the largest convex $\ell$-subgroup of $G$ that belongs to $Q$, called the quasi-torsion radical of $G$. Finite-valued $\ell$-groups form a torsion class $F_{v}$, and special-valued $\ell$-groups form a quasi-torsion class $S$.

We have shown that the finite-valued torsion radical of an $\ell$-group $G$ is the intersection of all maximal finite-valued subgroups of $G$ [6]. Let $S$ be the quasitorsion class of special-valued $\ell$-groups. We will show that the quasi-torsion radical $S(G)$ is the intersection of all the maximal special-valued subgroups of $G$. We will show that the class $A_{f}$ of almost finite-valued $\ell$-groups is a quasi-torsion class, and its quasi-torsion radical of $G$ is equal to the intersection of $G$ with the lateral completion of the finite-valued torsion radical of $G$ : $A_{f}(G)=F_{v}(G)^{L} \cap G$. Also for each $\ell$-group $G$, the following are equivalent:

1. There exists a largest finite-valued subgroup of $G$.

2. The set $\Delta$ of special values of $G$ is an $\ell$-ideal of $\Gamma(G)$.

3. $S(G)$ is the largest special-valued subgroup of $G$ and $S(G)$ is almost finitevalued.

\section{Maximal Special-Valued subgroups}

Definition 1.1. A special-valued subgroup of an $\ell$-group $G$ is an $\ell$-subgroup $U$ such that for each $0<g \in U, g=\vee_{\Lambda} g_{\lambda}$, where the $g_{\lambda}$ 's are disjoint and special in $G$. 
Thus if $g \in U$, then $|g|=\vee_{\Lambda} g_{\lambda}$, where $g_{\lambda} \in G$ are disjoint and special, but we don't require that $g_{\lambda} \in U$.

If $\cdots \subseteq C_{\alpha} \subseteq C_{\beta} \subseteq C_{\gamma} \subseteq \cdots$ is a chain of special-valued subgroups of $G$, then $\cup C_{\lambda}$ is a special-valued subgroup. Hence each special-valued subgroup is contained in a maximal special-valued subgroup.

Proposition 1.2. If $0<a=\vee_{A} a_{\alpha}$ and $0<b=\vee_{B} b_{\beta}$ are special-valued elements in $G$, where $A$ and $B$ are sets of values, then $a+b$ is special-valued in $G$ with the set of maximal elements in $A \cup B$ as special values.

Proof. Consider

$$
\begin{aligned}
0<a+b & =\vee_{A} a_{\alpha}+b=\vee_{A}\left(a_{\alpha}+b\right)=\vee_{A}\left(a_{\alpha}+\vee_{B} b_{\beta}\right) \\
& =\vee_{A}\left(\vee_{B}\left(a_{\alpha}+b_{\beta}\right)\right)=\vee_{A \cup B}\left(a_{\alpha}+b_{\beta}\right) .
\end{aligned}
$$

Suppose that $\alpha>\beta$, for some $\beta \in B$. Then $\alpha$ is greater than some subset of $B$ and disjoint from the other elements of $B$.

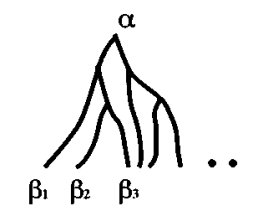

$$
\begin{aligned}
a_{\alpha} \wedge b & =a_{\alpha} \wedge\left(\vee_{B} b_{\beta}\right)=\vee_{B}\left(a_{\alpha} \wedge b_{\beta}\right) \\
& =\left(a_{\alpha} \wedge b_{\beta_{1}}\right) \vee\left(a_{\alpha} \wedge b_{\beta_{2}}\right) \vee\left(a_{\alpha} \wedge b_{\beta_{2}}\right) \vee \ldots \\
& =b_{\beta_{1}} \vee b_{\beta_{2}} \vee b_{\beta_{3}} \vee \ldots
\end{aligned}
$$

Thus $\left(a_{\alpha}+b_{\beta_{1}}\right) \vee\left(a_{\alpha}+b_{\beta_{2}}\right) \vee\left(a_{\alpha}+b_{\beta_{3}}\right) \vee \cdots=a_{\alpha}+\left(b_{\beta_{1}} \vee b_{\beta_{2}} \vee b_{\beta_{3}} \vee \ldots\right)$ is special with value $\alpha$, and $\alpha$ is maximal in $A \cup B$.

If $\alpha=\beta$, for some $\beta \in B$, then $a_{\alpha}+b_{\beta}$ is special with value $\alpha$, and $\alpha$ is maximal in $A \cup B$.

If $\alpha$ is not comparable with any $\beta$, then $a_{\alpha}+b_{\beta}=a_{\alpha} \vee b_{\beta}$ with values $\alpha$ and $\beta$, and both are maximal in $A \cup B$.

Corollary 1.3. If $a$ and $b$ are finite-valued, then so is $a+b$.

Proposition 1.4. If $b$ is a special-valued element with $b>a>0$, then $b+a$ is special-valued and has the same special values as $b$.

Proof. Suppose that $b=\vee_{\Lambda} b_{\lambda}$ with $b_{\lambda}$ disjoint and special. Then $a=b \wedge a=$ $\left(\vee_{\Lambda} b_{\lambda}\right) \wedge a=\vee_{\Lambda}\left(b_{\lambda} \wedge a\right)$. We have

$$
\begin{aligned}
b+a & =b+\vee_{\Lambda}\left(b_{\lambda} \wedge a\right) \\
& =\vee_{\Lambda}\left(b+\left(b_{\lambda}+a\right)\right) \\
& =\vee_{\Lambda}\left(\vee_{\Lambda} b_{\gamma}+\left(b_{\lambda} \wedge a\right)\right) \\
& =\vee_{\Lambda}\left(\vee_{\Lambda}\left(b_{\gamma}+\left(b_{\lambda} \wedge a\right)\right)\right) \\
& =\vee_{\gamma \in \Lambda, \lambda \in \Lambda}\left(b_{\gamma}+\left(b_{\lambda} \wedge a\right)\right) .
\end{aligned}
$$

If $\gamma \neq \lambda$, then $b_{\gamma}+\left(b_{\lambda} \wedge a\right)=b_{\gamma} \vee\left(b_{\lambda} \wedge a\right) \leq\left(b_{\gamma}+\left(b_{\gamma} \wedge a\right)\right) \vee\left(b_{\lambda}+\left(b_{\lambda} \wedge a\right)\right)$. Therefore $b+a=\vee_{\Lambda}\left(b_{\lambda}+\left(b_{\lambda} \wedge a\right)\right)$. 
Now we show that $b_{\lambda}+\left(b_{\lambda} \wedge a\right)$ is special with value $\lambda$. Since $b_{\lambda} \in G^{\lambda} \backslash G_{\lambda}$, where $G_{\lambda}$ is the regular subgroup of $G$, and $G^{\lambda}$ the cover of $G_{\lambda}$, we have $b_{\lambda}+\left(b_{\lambda} \wedge a\right) \in$ $G^{\lambda} \backslash G_{\lambda}$. Now let $\alpha$ be a value of $b_{\lambda}+\left(b_{\lambda} \wedge a\right)$. Then $b_{\lambda} \notin G_{\alpha}$, so $G_{\alpha} \subseteq G_{\lambda}$. If $G_{\alpha} \subset G_{\lambda}$, then $b_{\lambda} \leq b_{\lambda}+\left(b_{\lambda} \wedge a\right) \in G^{\alpha} \subseteq G_{\lambda}$. This contradicts the fact that $b_{\lambda} \in G^{\lambda} \backslash G_{\lambda}$. Therefore $\alpha=\lambda$.

Proposition 1.5. An $\ell$-subgroup $C$ is the largest special-valued subgroup of $G$ if and only if $C=S(G)$ which consists of all the special-valued elements of $G$.

Proof. ( $\Longleftarrow)$ If $C$ consists of all the special-valued elements of $G$, then it is the largest special-valued subgroup of $G$.

$(\Longrightarrow)$ If $0<g$ is special-valued, then the $\ell$-subgroup $\langle g\rangle$ of $G$ generated by $g$ is a special-valued subgroup of $G$. Therefore $\langle g\rangle \subseteq C$, and hence $C$ consists of all the special-valued elements of $G$.

If $b$ is special-valued and $b>a>0$, then by the last proposition, $b, b+a \in C$, so $a \in C$. Thus $C$ is convex and hence $C \subseteq S(G)$. Since $S(G)$ is a special-valued subgroup of $G, S(G) \subseteq C$.

Corollary 1.6. For an $\ell$-group $G$, the following are equivalent:

1. There exists a largest special-valued subgroup of $G$.

2. $S(G)$ consists of all the special-valued elements of $G$.

3. $b \in G$ is special and $b>a>0$ imply that $a$ is special-valued.

4. $S(G)$ contains all the special elements of $G$.

Proof. By the above proposition $1 \longleftrightarrow 2$, and clearly $2 \longrightarrow 3$.

$3 \longrightarrow 4 . b$ is special implies that $G(b) \subseteq S(G)$, so $S(G)$ contains all the special elements of $G$.

$4 \longrightarrow 2$. $0<g$ is special-valued implies that $g=\vee_{\Lambda} g_{\lambda}$. Each $g_{\lambda} \in S(G)$, and $S(G)$ is closed. Hence, we have $g \in S(G)$.

Proposition 1.7. Let $G$ be an $\ell$-group. If a is a positive special-valued element of $G, b$ is a negative element of $S(G)$, and $a+b$ is positive, then $a+b$ is special-valued in $G$.

Proof. $0<a=\vee_{A} a_{\alpha}$, where $a_{\alpha}$ are disjoint and special with value $\alpha$, and $0<$ $-b=\vee_{B}\left(-b_{\beta}\right)$, where $-b_{\beta}$ are disjoint and special with value $\beta$.

Now $0<a+b=\vee_{A} a_{\alpha}+b$, so $0<-b<\vee_{A} a_{\alpha}$, and hence by Proposition 1.4, $\left(\vee_{A} a_{\alpha}-b\right)$ is special-valued with the same set of special values as $\vee_{A} a_{\alpha}$. Thus $A$ is the set of special values for $\vee_{A} a_{\alpha}-b=\vee_{A} a_{\alpha}+\vee_{B}\left(-b_{\beta}\right)$. So each $\beta$ is less than or equal to one and only one $\alpha$ and is incomparable with the other $\alpha$ 's. Now consider

$$
0<a+b=\vee_{A} a_{\alpha}+b=\vee_{A}\left(a_{\alpha}+b\right)=\vee_{A}\left(\left(a_{\alpha}+b\right) \vee 0\right)
$$

Case I. $a_{\alpha} \wedge|b|=0$. In this case $\alpha$ is incomparable with all $\beta$, thus $\left(a_{\alpha}+b\right) \vee 0=$ $a_{\alpha}$.

Case II. $\alpha=\beta$ for some $\beta$. We then have

$$
0<-b=\vee_{B}\left(-b_{\beta}\right)=-b_{\beta}+\vee_{B \backslash\{\beta\}}\left(-b_{\gamma}\right),
$$

where $-b_{\beta}$ and $\vee_{B \backslash\{\beta\}}\left(-b_{\gamma}\right)$ are disjoint. Hence

$$
\left(a_{\alpha}+b\right) \vee 0=\left(a_{\alpha}+b_{\beta}-\vee_{B \backslash\{\beta\}}\left(-b_{\gamma}\right)\right) \vee 0=\left(a_{\alpha}+b_{\beta}\right) \vee 0
$$

is a positive element in $S(G)$ with all special values less than or equal to $\alpha$, since $a_{\alpha}+b_{\beta}$ is disjoint from $\vee_{B \backslash\{\beta\}}\left(-b_{\gamma}\right)$. 
Case III. $\alpha>\beta$ for some $\beta$. Let $B_{\alpha}=\{\beta \in B \mid \alpha>\beta\}$. Hence for $\beta \in B \backslash B_{\alpha}$, $\alpha \| \beta$.

We now have that $-b=\vee_{B}\left(-b_{\beta}\right)$ and that $a_{\alpha} \wedge(-b)=a_{\alpha} \wedge \vee_{B}\left(-b_{\beta}\right)=$ $\vee_{B}\left(a_{\alpha} \wedge\left(-b_{\beta}\right)\right)=\vee_{B_{\alpha}}\left(-b_{\beta}\right)$, so $-b=\vee_{B_{\alpha}}\left(-b_{\beta}\right)+\vee_{B \backslash B_{\alpha}}\left(-b_{\beta}\right)$ and $a_{\alpha}+b=$ $a_{\alpha}-\vee_{B_{\alpha}}\left(-b_{\beta}\right)-\vee_{B \backslash B_{\alpha}}\left(-b_{\beta}\right)$, where $a_{\alpha}-\vee_{B_{\alpha}}\left(-b_{\beta}\right)$ and $\vee_{B \backslash B_{\alpha}}\left(-b_{\beta}\right)$ are positive and disjoint. Therefore, $\left(a_{\alpha}+b\right) \vee 0=a_{\alpha}-\vee_{B_{\alpha}}\left(-b_{\beta}\right)$ is special with value $\alpha$.

Thus $\left(a_{\alpha}+b\right) \vee 0$ can be written as the join of disjoint special elements and has all its special values less than or equal to $\alpha$. Hence $0<a+b=\vee_{A}\left(\left(a_{\alpha}+b\right) \vee 0\right)$ can be written as the join of disjoint special elements, hence is special valued.

Proposition 1.8. If $U$ is a special-valued subgroup of $G$, then $U+S(G)$ is a specialvalued subgroup of $G$.

Proof. Since $S(G)$ is an $\ell$-ideal, we have that $U+S(G)$ is an $\ell$-subgroup of $G$. Consider $0<g=a+b \in U+S(G)$, with $a \in U$ and $b \in S(G)$. We have $g+S(G)=a+S(G)=a \vee 0+S(G)$, so without loss of generality, we may assume $a>0.0<a+b=a+b^{+}-b^{-}$, where $a+b^{+}$can be written as the join of disjoint special elements of $G$. So by Proposition 1.7, $a+b$ can be written as the join of disjoint special elements of $G$.

We are now ready to describe the special-valued quasi-torsion radical for an $\ell$-group by its maximal special-valued subgroups.

Theorem 1.9. $S(G)$ is the intersection of all the maximal special-valued subgroups of $G$.

Proof. By Proposition 1.8, $S(G)$ is contained in the intersection of all the maximal special-valued subgroups of $G$. Now we pick $0<a \in G \backslash S(G)$. We need to show there exists a maximal special-valued subgroup that does not contain $a$. If $a$ is not special-valued, then it does not belong to any special-valued subgroups of $G$. Now suppose that $a=a_{1} \vee a_{2} \vee a_{3} \vee \cdots$, where $a_{i} \in G$ are disjoint and special. $G(a) \not \subseteq S(G)$, so there exists $b \in G$ such that $a>b>0$ and $b$ is not special-valued in $G$. By Proposition 1.4, $a+b$ is special-valued. Thus there exists a maximal special-valued subgroup of $G$ that contains $a+b$ but not $a$.

Proposition 1.10. If $0<a$ and $0<b$ are special-valued elements in an $\ell$-group $G$, then so are $a \wedge b$ and $a \vee b$.

Proof. Consider $0<\vee_{A} a_{\alpha}$ and $0<\vee_{B} b_{\beta}$, where the $a_{\alpha}$ 's are disjoint and $a_{\alpha}$ has special value $\alpha$, and the $b_{\beta}$ 's are disjoint and $b_{\beta}$ has special value $\beta$.

Let $C=\{\alpha \in A \mid$ there exists $\beta \in B$ comparable with $\alpha\}$. Then $C=C_{1} \cup C_{2}$, where $C_{1}=\{\alpha \in C \mid \beta \leq \alpha$ for some $\beta \in B\}$ and $C_{2}=\{\alpha \in C \mid \alpha<\beta$ for some $\beta \in B\}$.

We have

$$
\begin{aligned}
a \vee b & =\left(\vee_{A} a_{\alpha}\right) \vee\left(\vee_{B} b_{\beta}\right)=\vee_{A}\left(a_{\alpha} \vee\left(\vee_{B} b_{\beta}\right)\right)=\vee_{A}\left(\vee_{B}\left(a_{\alpha} \vee b_{\beta}\right)\right) \\
& =\vee_{A \cup B}\left(a_{\alpha} \vee b_{\beta}\right)=\vee_{A \backslash C} a_{\alpha} \vee_{C}\left(a_{\alpha} \vee b_{\beta}\right) \vee_{B \backslash C} b_{\beta} \\
& =\vee_{A \backslash C} a_{\alpha} \vee\left(a_{\alpha} \vee\left(\vee_{C_{1}} b_{\beta}\right)\right) \vee\left(b_{\beta} \vee\left(\vee_{C_{2}} a_{\alpha}\right)\right) \vee_{B \backslash C} b_{\beta} .
\end{aligned}
$$

Therefore, $a \vee b$ is special-valued with values maximal in $A \cup B$. Similarly,

$$
a \wedge b=\left(\vee_{A} a_{\alpha}\right) \wedge\left(\vee_{B} b_{\beta}\right)=\vee_{A}\left(a_{\alpha} \wedge\left(\vee_{B} b_{\beta}\right)\right)=\vee_{A \cup B}\left(a_{\alpha} \wedge b_{\beta}\right)=\vee_{C}\left(a_{\alpha} \wedge b_{\beta}\right) .
$$

Therefore, $a \wedge b$ is special-valued with values minimal in $C$. 
Theorem 1.11. A maximal special-valued subgroup $U$ of an abelian $\ell$-group $G$ is weakly saturated; i.e., special components of elements of $U$ belong to $U$.

Proof. Suppose that $v \in U$. Then $v=g_{1} \vee g_{2} \vee g_{3} \vee \ldots$, where $g_{i} \in G$ are disjoint and special. Assume (by way of contradiction) that $g_{1} \notin U$, and let $\left\langle U, g_{1}\right\rangle$ be the $\ell$-subgroup of $G$ generated by $U$ and $g_{1}$. Consider $0<f \in\left\langle U, g_{1}\right\rangle$. Then

$$
f=f \vee 0=\vee_{I} \wedge_{J}\left(\left(u_{i j} \pm n_{i j} g_{1}\right) \vee 0\right)
$$

where $I$ and $J$ are finite index sets, $u_{i j} \in U$ and $n_{i j}$ are positive integers. We will first show that for fixed $i$ and $j$, each $\left(u_{i j} \pm n_{i j} g_{1}\right) \vee 0$ is special-valued.

Consider $\left(u \pm n g_{1}\right) \vee 0, u \in U$ hence $|u|=h_{1} \vee h_{2} \vee h_{3} \vee \ldots$, where $h_{i} \in G$ are disjoint and special.

If the value of $g_{1}$ is different from all those of $u$, then clearly $\left(u \pm n g_{1}\right) \vee 0$, is special-valued.

If the value of $g_{1}$ is the same as a value of $u$, say, a value of $h_{1}$, but the value of $g_{1}$ is the same as that of $h_{1} \pm n g_{1}$, then we have $\left(u \pm n g_{1}\right) \vee 0$ is special-valued.

Now consider the case when the value of $g_{1}$ is the same as that of $h_{1}$, and values of $h_{1} \pm n g_{1}$ are less than that of $g_{1}$. We then have

$$
\left(h_{1} \pm n g_{1}\right) \vee 0 \leq(u \pm n v) \vee 0=f_{1} \vee f_{2} \vee f_{3} \vee \ldots
$$

where $0<f_{i} \in G$ are disjoint and special, since $(u \pm n v) \vee 0 \in U$.

Let $F=\left\{f_{j} \mid f_{j} \leq g_{1}\right\}, \vee\left\{f_{j} \mid f_{j} \in F\right\}$ exists in $G$ because $\vee\left\{f_{j} \mid f_{j} \in F\right\}=$ $\left(f_{1} \vee f_{2} \vee f_{3} \vee \ldots\right) \wedge g_{1}$. We claim that

$$
\left(h_{1} \pm n g_{1}\right) \vee 0=\vee\left\{f_{j} \mid f_{j} \in F\right\} .
$$

Clearly $\left(h_{1} \pm n g_{1}\right) \vee 0 \leq \vee\left\{f_{j} \mid f_{j} \in F\right\}$, since $\left(h_{1} \pm n v\right) \vee 0 \leq f_{1} \vee f_{2} \vee f_{3} \vee \ldots$ On the other hand, each $f_{j} \leq\left(h_{1} \pm n g_{1}\right) \vee 0$ implies that $\vee\left\{f_{j} \mid f_{j} \in F\right\} \leq\left(h_{1} \pm n g_{1}\right) \vee 0$. We have shown that $\left(h_{1} \pm n g_{1}\right) \vee 0$ is special-valued which implies that $\left(u \pm n g_{1}\right) \vee 0$ is special-valued. Thus by Proposition 1.10, $\vee_{I} \wedge_{J}\left(\left(u_{i j} \pm n_{i j} g_{1}\right) \vee 0\right)$ is special-valued in $G$.

Corollary 1.12. A maximal special-valued subgroup $U$ of an abelian $\ell$-group is special-valued as an $\ell$-group.

Proof. Let $U$ be a maximal special-valued subgroup of $G$. For each $0<u \in U$, $u=g_{1} \vee g_{2} \vee g_{3} \vee \ldots$, where $g_{i} \in G$ are disjoint and special. By Theorem 1.11, each $g_{i} \in U$. Also each $g_{i}$ is special in $U$. Therefore $U$ is special-valued as an $\ell$-group.

\section{Almost finite-VAlued $\ell$-Groups}

Definition 2.1. An $\ell$-group $G$ is almost finite-valued if it is special-valued and the principal convex $\ell$-subgroup $G(g)$ is finite-valued for each finite-valued element $g \in G$.

We use $A_{f}$ to indicate the class of almost finite-valued $\ell$-groups. We first list some basic properties of $A_{f}$ and brief proofs.

1. $F_{v} \subseteq A_{f} \subseteq S$, where $F_{v}$ is the torsion class of finite-valued $\ell$-groups, and $S$ is the quasi-torsion class of special-valued $\ell$-groups.

2. $A_{f}$ is closed with respect to products. 
Proof. The quasi-torsion class $S$ of special-valued $\ell$-groups is closed with respect to products, and if $0<g$ is special in a product, then it is special in one of the factors, so $G(g)$ is finite-valued.

3. $A_{f}$ is not closed with respect to $\ell$-subgroups.

For example, for each $x \in[0,1]$, let $R_{x}$ be the real numbers. Then $\prod_{x \in[0,1]} R_{x} \in A_{f}$, but $C[0,1] \notin S$.

4. $G \in A_{f}$ does not imply that $G^{L} \in A_{f}$.

For example, let $\Lambda=\bigwedge$... Then $\Sigma(\Lambda, R) \in A_{f}$, but $\Sigma(\Lambda, R)^{L}=$ $V(\Lambda, R) \notin A_{f}$.

5. If $G \in A_{f}$, then $F_{v}(G)$ is the $\ell$-ideal generated by all the $G(g)$ with $g$ special. So $F_{v}(G)$ is the largest finite-valued subgroup of $G$.

6. A laterally complete special-valued $\ell$-group is almost finite-valued if and only if its set of special values $\Delta$ contains no copy of

$$
\Lambda=\bigwedge \ldots .
$$

Proof. Suppose $\Lambda$ is contained in $\Delta$. Then let $g$ be a special element with value $\delta$ which is the maximal element of $\Delta$. Since $G$ is laterally complete, $G(g)$ contains an element with an infinite number of values. On the other hand, let $g$ be a special element with value $\delta$, and $\Delta$ contains no copy of $\Lambda$. Then the ideal $\bar{\delta}=\{\alpha \in \Delta \mid \alpha \leq \delta\}$ contains only a finite number of roots, so $G(g)$ is finite-valued.

7. For a completely distributive $\ell$-group $G$, the following are equivalent:

(a) $G^{L}$ is almost finite-valued.

(b) The plenary set $\Delta$ of all essential values of $\Gamma(G)$ contains no copy of the set $\Lambda$ of (6).

Proof. By [3], $\Delta \cong$ the set of all the special elements of $\Gamma\left(G^{L}\right)$.

8. If $G$ is a special-valued vector lattice, then without loss of generality, we assume that $\Sigma(\Delta, R) \subseteq G \subseteq V(\Delta, R) . \quad V(\Delta, R) \in A_{f}$ if and only if $\Delta$ contains no copy of $\Lambda$ as in (6). Thus each abelian $a^{*}$-extension of $G$ is almost finite-valued if and only if $\Delta$ contains no copy of $\Lambda$.

Theorem 2.2. For an $\ell$-group $G$, the following are equivalent:

1. There exists a largest finite-valued subgroup of $G$.

2. $F_{v}(G)$ consists of all the finite-valued elements of $G$.

3. $0<a<b$ and $b$ is special imply that $a$ is finite-valued.

4. $b$ is special implies that $G(b)$ is finite-valued.

5. $F_{v}(G)$ contains all the special elements of $G$.

6. The set $\Delta$ of special values of $G$ is an ideal of $\Gamma(G)$.

7. $S(G)$ is the largest special-valued subgroup of $G$, and $S(G)$ is almost finitevalued.

Proof. We proved in [6] that 1,2 , and 3 are equivalent, and clearly $3 \longleftrightarrow 4$ and $2 \longleftrightarrow 5$.

Now suppose $0<g \in G$ is special with value $G_{\alpha}$. Then $G_{\alpha} \longrightarrow G_{\alpha} \cap G(g)$ is a one-to-one order-preserving map of the regular subgroups of $G$ that do not contain $G(g)$ onto the regular subgroups of $G(g)$. 
$6 \longrightarrow 4 . \Delta$ is an $\ell$-ideal of $\Gamma(G)$ implies that all the regular subgroups of $G(g)$ are special if $g$ is special. So $G(g)$ is finite-valued.

$4 \longrightarrow 7$. $G(g)$ is finite-valued implies that $G(g) \subseteq S(G)$. So $S(G)$ contains all the special elements.

$7 \longrightarrow 4 . \quad g$ is special implies $g \in S(G)$. Thus $G(g) \subseteq S(G)$, hence $G(g)$ is finite-valued.

$4 \longrightarrow 6$ is clear.

This theorem shows that if $G$ has a largest finite-valued subgroup, then $G$ has a largest special-valued subgroup, although the converse is not true.

For example, let $\Lambda=\bigwedge$....

$G=V(\Lambda, R) \in S$, but $G$ has no largest finite-valued subgroup.

Actually, there exists a largest special-valued subgroup of $G$ if and only if for each special $b \in G, G(b)$ is special-valued. In turn, this is equivalent to the assertion that for each special value $\delta \in \Delta$, the ideal $\bar{\delta}=\{\gamma \in \Gamma(G) \mid \gamma \leq \delta\}$ contains a dual ideal of special elements with zero intersection.

We now give the proof that $A_{f}$ is a quasi-torsion class.

Theorem 2.3. The almost finite-valued $\ell$-groups form a quasi-torsion class $A_{f}$.

Proof. $G$ is almost finite-valued if and only if the set $\Delta$ of special values is a plenary subset and an ideal of $\Gamma(G)$. A special-valued $\ell$-group $G$ is almost finite-valued if and only if $\Delta$ is an ideal of $\Gamma(G)$.

1. $A_{f}$ is closed with respect to convex $\ell$-subgroups.

Suppose $C$ is a convex $\ell$-subgroup of an almost finite-valued $\ell$-group $G$. Then $C \in S$, since $S$ is a quasi-torsion class. The set $C_{\Delta}$ of all special values of $C$ is an ideal and a plenary subset of $G_{\Delta}$, the set of all special values of $G$, and $G_{\Delta}$ is an ideal in $\Gamma(G)$. Therefore $C_{\Delta}$ is an ideal in $\Gamma(C)$ and a plenary subset of $\Gamma(C)$.

2. $A_{f}$ is closed with respect to complete $\ell$-homomorphic images.

Let $C$ be a closed $\ell$-ideal of $G \in A_{f}$. Then $G / C \in S$. Suppose $C+g$ is special in $G / C, g=g_{1} \vee g_{2} \vee g_{3} \vee \ldots$ in $G$, with not all $g_{i}$ belonging to $C$. If there is more than one component of $g$ not in $C$, say, $g_{1}$ is one of them, then $C+g_{1}$ is a proper component of $C+g$, so $C+g$ is not special. So without loss of generality, $g$ is special in $G$. The convex $\ell$-subgroup of $G / C$ generated by $C+g$ is isomorphic to $\frac{G(g)}{C \cap G(g)}$, and it is finite-valued, since $F_{v}$ is a torsion class.

3. $A_{f}$ is closed with respect to joins of convex $\ell$-subgroups.

$S(G)$ is the largest convex $\ell$-subgroup that belongs to $S$. If the largest convex $\ell$-subgroup of $G$ that is almost finite-valued exists, then it is an $\ell$-ideal of $S(G)$. So without loss of generality, we assume that $G$ is special-valued. A convex $\ell$-subgroup $C$ of $G$ is almost finite-valued if and only if $C_{\Delta}$ is an ideal of $\Gamma(C)$, but $\Gamma(C)$ is an ideal of $\Gamma(G)$. So $C$ is almost finite-valued if and only if $C_{\Delta}$ is an ideal in $\Gamma(G)$.

If $C_{1} \subseteq C_{2} \subseteq C_{3} \subseteq \ldots$ form a chain of convex $\ell$-subgroups of $G$ that belong to $A_{f}$, then their sets of special values $C_{1 \Delta} \subseteq C_{2 \Delta} \subseteq C_{3 \Delta} \subseteq \ldots$ form a chain of ideals in $\Gamma(G)$, so $\cup C_{i \Delta}$ is an ideal in $\Gamma(G)$, hence $\cup C_{i}$ is almost finite-valued.

If $B$ and $C$ are almost finite-valued, and $0<g \in B+C$ is special with value $\delta$, then $g \in B$ or $g \in C$. In fact, $g$ is equal to the sum of positive elements from $B \cup C$, and one of them must have value $\delta$. Without loss of generality, there exists $0<b \in B$ with a value $\delta$, hence $n b>g$ for some $n \in N$, so $g \in B$. Thus $B(g)$ is 
finite-valued, which implies $(B+C)(g)$ is finite-valued. Therefore, $B+C$ is almost finite-valued.

The torsion radical $F_{v}(G)$ is a characteristic $\ell$-ideal of $G$, and $F_{v}(G) \subseteq G \subseteq G^{L}$, where $G^{L}$ is the lateral completion of $G$. Actually, $F_{v}(G)^{L} \subseteq G^{L}$. This follows from the following proposition.

Proposition 2.4. $C \in \mathcal{C}(G)$, the set of all convex $\ell$-subgroups of $G$, implies that $C^{L} \subseteq G^{L}$.

Proof. $C \subseteq C^{\prime \prime}$, the polar of $C$ in $G^{L}$, and since $C^{\prime \prime}$ is closed, it is laterally complete. Now $G$ is dense in $G^{L}$, so $0<x \in C^{\prime \prime} \subseteq G^{L}$ implies $x>y>0$, for some $y \in G \cap C^{\prime \prime}$. In particular, $y$ is not disjoint from $C^{+}$, so $y \wedge c>0$, for some $c \in C$. Therefore $x>y \wedge c \in C$, so $C$ is dense in the laterally complete $\ell$-group $C^{\prime \prime}$, and hence $C^{L} \subseteq C^{\prime \prime} \subseteq G^{L}$.

The following theorem demonstrates the relation between the torsion radicals $A_{f}(G)$ and $F_{v}(G)$.

Theorem 2.5. $A_{f}(G)=F_{v}(G)^{L} \cap G$.

Proof. $F_{v}(G)^{L}$ is special-valued and has the same set $\Delta$ of special values as $F_{v}(G)$ by the result in [3]. Consider $0<g \in F_{v}(G)^{L} \cap G, g=h_{1} \vee h_{2} \vee h_{3} \vee \cdots$, where $h_{i}$ are disjoint and special in $F_{v}(G)^{L}$. Now there exists a special element $0<g_{1} \in F_{v}(G)$ with the same value as $h_{1}$. Thus $n g_{1}>h_{1}$ for some $n \in N$, so $h_{1}=n g_{1} \wedge g \in F_{v}(G)$; thus $G\left(h_{1}\right)$ is finite-valued.

Suppose $0<u<g$ with $u \in G$. Then $u=u \wedge g=u \wedge\left(\vee h_{i}\right)=\vee\left(u \wedge h_{i}\right)$. Each $u \wedge h_{i} \in G\left(h_{i}\right)$ is finite-valued. Therefore $u$ is special-valued.

If $0<a \in G(g)$ is special, then $a<n g$, so $a<n h_{i}$, for some $n \in N$. Thus $G(a)$ is finite-valued. Therefore, $G(g) \subseteq A_{f}(G)$, so $F_{v}(G)^{L} \cap G \subseteq A_{f}(G)$.

Conversely, consider $0<g \in A_{f}(G)$. Then $g=g_{1} \vee g_{2} \vee g_{3} \vee \cdots$, where $g_{i}$ are disjoint and special, and each $G\left(g_{i}\right)$ is finite-valued. Therefore each $g_{i} \in F_{v}(G)$, so $g \in F_{v}(G)^{L} \cap G$.

Thus if $G$ is laterally complete, then $A_{f}(G)=F_{v}(G)^{L}$. So $G \in A_{f}$ if and only if $G=F_{v}(G)^{L}$. In fact, the proof of the theorem shows that $F_{v}(G)(1) \cap G=A_{f}(G)$, where $F_{v}(G)(1)$ is the $\ell$-group generated by the joins of disjoint elements from $F_{v}(G)$ [9]. Therefore $F(G)(1) \cap G=F_{v}(G)^{L} \cap G$. We now discuss almost finite-valued $\ell$-groups that are laterally complete.

Proposition 2.6. Let $G$ be a special-valued laterally complete $\ell$-group. If $g \in G$ is infinite valued, then $F_{v}(G)+g$ is not finite-valued in $G / F_{v}(G)$.

Proof. If $F_{v}(G)+g$ is finite-valued, then without loss of generality, it is special in $G / F_{v}(G)$. But $g$ can be written as $a+b$ with $a \wedge b=0$, where $a$ and $b$ are both infinite-valued. Thus $F_{v}(G)+a$ and $F_{v}(G)+b$ are non-zero components of $F_{v}(G)+g$. This contradicts the fact that $F_{v}(G)+g$ is special in $G / F_{v}(G)$.

Proposition 2.7. Let $G$ be a special-valued laterally complete $\ell$-group. $G \in A_{f}$ if and only if $G / F_{v}(G)$ contains no special elements.

Proof. ( $\Longrightarrow)$ If $g \in G \backslash F_{v}(G)$, then $g$ is infinite-valued and hence $F_{v}(G)+g$ is not special. 
$(\Longleftarrow)$ If $0<g \in G$ is special, then $g \in F_{v}(G)$. For otherwise $F_{v}(G)+g$ is not special in $G / F_{v}(G)$. Therefore $F_{v}(G)$ contains all the special elements of $G$, and hence $G \in A_{f}$.

The next theorem follows from the above theorem and propositions.

Theorem 2.8. For a special-valued laterally complete $\ell$-group $G$, the following are equivalent:

1. $G \in A_{f}$.

2. $G=F_{v}(G)^{L}$.

3. $F_{v}(G)$ contains all the special elements of $G$.

4. The set of special values of $G$ contains no copy of $\bigwedge$....

In particular, this theorem holds for the $\ell$-group $V(\Delta, R)$, where $\Delta$ is any root system.

\section{REFERENCES}

1. M. Anderson, P. Bixler, and P. Conrad, Vector lattices with no proper a-subspaces. Archiv Math. (basel), 41 (1983), 427-433. MR 85h:06038

2. M. Anderson, and T. Feil, Lattice-Ordered Groups: An Introduction. D. Reidel, 1987. MR 90b:06001

3. P. Bixler, and M. Darnel, Special-valued $\ell$-groups. Algebra Universalis, 22 (1986), 172-191. MR 88h:06021

4. R. Byrd, Lattice-ordered groups. Ph.D Dissertation. Tulane University, 1966.

5. Y. Chen, Torsion classes of lattice-ordered groups and special-valued subgroups. Ph.D dissertation, University of Kansas, 1992.

6. Y. Chen, P. Conrad, and M. Darnel, Finite-valued subgroups of lattice-ordered groups. Czech Math Journal, 46(121) 1996. CMP 97:01

7. P. Conrad, J. Harvey, and W.C. Holland, The Hahn embedding theorem for lattice-ordered groups. Trans. Amer. Math. Soc., 108 (1963), 143-149. MR 27:1519

8. M. Darnel, Theory of Lattice-Ordered Groups. Marcel Dekker, Inc, 1994. MR 95k:06032

9. J. Martinez, Torsion theory for lattice-ordered groups. Czech. Math. J., 25 (1975), 284-299. MR 52:10536

Department of Mathematical Sciences, Central Connecticut State University, New Britain, Connecticut 06050

E-mail address: chen@ccsua.ctstateu.edu

Department of Mathematics, University of Kansas, Lawrence, Kansas 66045 